\title{
A Collage image Creation \& "Kanisei" ANALYSIS SySTEM By COMBINING MULTIPLE IMAGES
}

\author{
Anju Kawamoto ${ }^{1}$, Yasuhiro Hayashi ${ }^{2}$, Izumi Fuse $^{3}$ and Yasushi Kiyoki ${ }^{1}$ \\ ${ }^{1}$ Faculty of Environmental Information, Keio University \\ 5322, Endo, Fujisawa, Kanagawa, 252-0816, Japan \\ ${ }^{2}$ Graduate School of Media and Governance, Keio University \\ 5322, Endo, Fujisawa, Kanagawa, 252-0816, Japan \\ ${ }^{3}$ Information Initiative Center, Hokkaido University \\ Nishi 5, Kita 11, Kita-ku, Sapporo, Hokkaido, 060-0811, Japan
}

\begin{abstract}
The collage is an artistic method to create a new image by combining multiple images under some rules or conditions for collage creators. To realize a mechanism to interpret "Kansei" of the collage creator by the combination process of multiple image-materials and a formed collage image, we propose a new system to analyze the collage work by using a database stored to each collage creation information. The collage works which a creator made by using this system was evaluated. And also, we clarified how to combine image-materials to make the good collage image having specific impression.
\end{abstract}

\section{KEYWORDS}

Collage Image, Image Creation, Semantic Analysis, Image Processing, Image Database

\section{INTRODUCTION}

The collage is an artistic method to create a new image by combining multiple images under some rules or conditions for collage creators. The outline of the collage is shown in Figure 1.

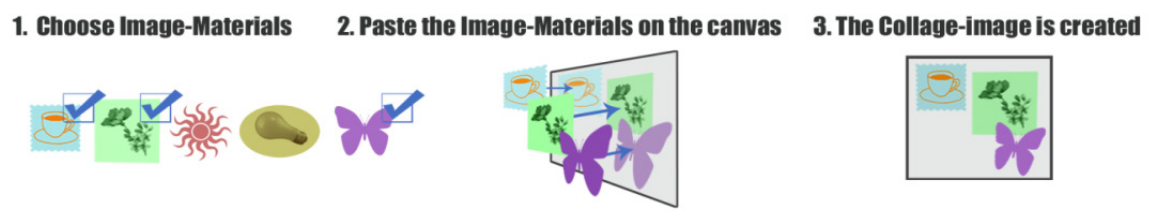

Figure 1. The Outline of the Collage

The creator chooses image martials for make a collage image. Then, the creator arranges images on the canvas while the creator considers position and size of each image. Finally, the collage image is made.

The purpose of the collage creation is to understand "Kansei" of human by combining process of the images as graphic materials to create and a combined image. The concept of "Kansei" includes several meanings on sensitive recognition, such as human senses, feelings, sensitivity and psychological reactions. 
The International Journal of Multimedia \& Its Applications (IJMA) Vol.9, No.1, February 2017

The collage has been already used in various fields. In the real world, it is applied to the artistic expression[1] and the psychoanalysis [2,3]. People enjoy humor of the combined image which do not exist in the real world and also they perform the self-understanding or stress emission through a series of processes for forming a collage image. The collage has been also utilized in the information retrieval research field [4]. In this field, Query-by-multiple-image is one of methods to retrieve images suited to retrievers' intention [5].

In this method, the retriever combines images his/her own have and creates an image-query for searching image data by the image. The retrieval method can create the image-query easily in comparison with the drawing method for CBIR (Content-based Image Retrieval)[6, 7].

Our goal is to realize a mechanism to understand "Kansei" by the combination process of the material images and a formed collage image on computers.

In the collage technique as previous research, psychologists have considered that facets of something of a creator are included to the collage work. For this reason, following points are important in order to interpret "Kansei" and intention of human.

- What kind of psychological meanings of a collage creator do the image-materials to be selected in the collage work have?

- How is the mental symptom expressed as combination of images?

- However, the relevance between image-materials of collage and "Kansei" has not been fully researched because the collage technique is not able to solve the following points [8]:

- The format to analyze a collage work

- Characteristic expression and meanings in a collage work

From the viewpoint of this among "Kansei" analysis, we have already proposed an imagematerial selection method for the collage work by using images and "Kansei" databases. In this method, we had examined the relevance between the image-materials and the creator's conscious mind. By the results of this examination, we got knowledge as many collage creators choose image-materials to make the collage even if they dislike them. The collage image is not only combined images the collage creator likes but also new image including semantic values.

In this paper, we propose a new system to analyze the collage work by using a database stored to collage creation information. Each image material used for creation collage corresponds with atoms of chemical substances. We explore meanings of the collage image that consists of atomic images. This operation is like analyzing molecular combination. Our system was developed and we evaluated collage works which a user made by using this system. And also, we clarified how to combine image-materials to make the collage having specific impression.

\section{A Collage Image Creation \& "Kanisei" Analysis System By Combining Multiple ImageS}

The system enables the "Kansei" analysis of an image formed by the combination of multiple images as the collage image. The features of this system are shown as follows:

1. This system is able to analyze the relevance of each impression of image-materials and whole impression of the combined image as collage.

2. This system is also able to analyze the relevance of attributes of a collage creator and an impression of the combined image as collage. 
The International Journal of Multimedia \& Its Applications (IJMA) Vol.9, No.1, February 2017

The system consists of a database, an image editing tool and several functions to analyze the collage work. The target users of this system are a collage analyst as the administrator and a collage creator as the user. The collage analyst sets the image-materials for making collage images in the collage image database and analyze the created collage images by this system. The collage creator selects the image-materials that he/she wants to use to compose the collage image on the image editing tool.

\subsection{Definitions Of The Collage Database Schema}

The schema of the collage database is defined as follows. A kind of data stored in the database are the image-materials, the collage-image combined the image-materials, and procedures and parameters of the image processing that correspond with the collage creators' intentions. In this system, impression of each image-material and the collage image is managed independently. These data are stored to each table having following structures.

\subsubsection{The IMAGE-MATERIAL TABLE}

The information about the image-material is recorded to the image-material table. The details of the image-material table are shown in Table 1.

Table 1. The Image-Material Table

\begin{tabular}{|l|l|l|}
\hline \multicolumn{1}{|c|}{ Property } & \multicolumn{1}{c|}{ Description } & \multicolumn{1}{c|}{ Data Type } \\
\hline m_id & Image-Material ID & INTEGER \\
\hline m_url & Image-Material URL & TEXT \\
\hline format & Flag for Distinguishing Photo whether or not & BOOLEAN \\
\hline genre & Genre of Image-Material & TEXT \\
\hline warm & Degree of Impression about 'warm' & INTEGER \\
\hline dynamic & Degree of Impression about 'dynamic' & INTEGER \\
\hline happy & Degree of Impression about 'happy' & INTEGER \\
\hline light & Degree of Impression about 'light' & INTEGER \\
\hline
\end{tabular}

Genre, format, impressions and data of the image-material are stored in this table. As the genre, this system handles 5 types genres ('nature', 'food', 'human', 'animal', 'symbol') based on the results of the previous research. The format of the image-material is stored into the format column as a binary value. If the format is picture, the stored value is expressed as 'true'.

This system deals with four basic impressions ('warm', 'dynamic', 'happy', 'light'). If the numerical value of each basic impression is 0 , it means that an image is unrelated to the word of the basic impression. If the numerical value is positive, then it means that an image is related to the word. On the other hand, if the numerical value is negative, then it means that an image has the opposite impression ('cold', 'quiet', 'sad', 'heavy') to each impression word. These five basic impression words were extracted from the impression word group for searching for paintings proposed by Kurita, Kato (1993)[9].Moreover, we had added the impression words that suitable for the collage.

\subsubsection{The Collage Image TABLE}

The information of the collage image and profile of a collage creator is stored to the collage image table. The structure of the collage image table is shown in Table 2. 
Table 2. The Collage Image Table

\begin{tabular}{|l|l|l|}
\hline \multicolumn{1}{|c|}{ Property } & \multicolumn{1}{c|}{ Description } & \multicolumn{1}{c|}{ Data Type } \\
\hline c_id & Collage Image ID & INTEGER \\
\hline c_url & Collage Image URL & TEXT \\
\hline name & Name of Creator & INTEGER \\
\hline gender & Gender of Creator & CHAR(1) \\
\hline age & Age of Creator & INTEGER \\
\hline c_imp & Impression of Collage Image & TEXT \\
\hline rule & Characteristic Representation & TEXT \\
\hline
\end{tabular}

The entire impression of the collage work is stored to 'c_imp' column as text type. The gender of the collage creator is stored into 'c_gender' column as M or F. The characteristic representation on the collage image is recorded to 'rule' column as text data.

\subsubsection{The Image Processing Table}

A series of procedures and parameters of image processing for creating a collage image by combination of multiple images as many record data are saved into this table. The details of the image processing table are shown in Table 3.

Table 3. The Image Processing Table

\begin{tabular}{|l|l|l|}
\hline \multicolumn{1}{|c|}{ Property } & \multicolumn{1}{c|}{ Description } & \multicolumn{1}{c|}{ Data Type } \\
\hline $\mathrm{m}$ id & Image Material ID & INTEGER \\
\hline c_id & Collage Image ID & INTEGER \\
\hline manipulation & Manipulation Class of Image Processing & TEXT \\
\hline parameter & Parameter for Manipulation & TEXT \\
\hline
\end{tabular}

Each record on the image processing table means that a collage image includes an image-material that is edited by a parameter. The system provides four functions ('zoom', 'layer', 'rotation', 'position') of the image processing. Selected image processing is marked to the 'manipulation' column as text data. The zoom means the magnification ratio and the ratio is stored as the parameter. The hierarchy order of each image on the canvas is stored as the parameter of the layer. We regard the magnification ratio and the order of the image layer as emphasized impression. The rotation angle is also stored as the parameter of the rotation. The parameter of the position corresponds to $\mathrm{x}$ and y coordinate of a collage image. All parameters are described as text type in the database to store different types of each parameter.

\subsection{A Construction Method of the Collage Database for Creation and ANALYSIS}

The construction method of the collage database is shown as follows.

\section{Step 1. Collecting The Image-Materials}

The image-materials being used to create the collage image are prepared by the administrator of this system in advance. The administrator needs to keep impartial impression of the prepared image-materials. An image that strongly has a specific impression is not suited as the image material because there is potential for bias in the use frequency of the image-materials. 
The International Journal of Multimedia \& Its Applications (IJMA) Vol.9, No.1, February 2017

\section{Step 2. Storing The Image-Materials to The Collage Database}

The administrator distinguishes elements of each image-material and stores them into the imagematerial table in the collage database. Especially, the impression words of each image are defined by the administrator while referring to the results of a questionnaire that is related to relevance between an image and impression. The degree of impression is represented as an integer value from -2 to 2 .

\section{Step 3. Creating The Collage Image and Storing It into The Collage Database}

The collage creator edits the image-materials to create the collage image by using the image editing tool. The created collage image and its entire impression are stored to the collage image table on the image database.

Step 4. Storing The Procedures and Parameters of the Image Processing to The collage database

The procedures and parameters of the image processing that the collage creator uses the image editing tool for are stored to the image processing table on the image database.

\subsection{Implementation Method Of The "Kansei" Analysis System For The COLlage IMAges}

In order to develop our system, PostgreSQL is used for the database system. Additionally, as an image editing tool, we utilized a web-based application for the image processing that is developed by Fuse, Okabe and Makino [10]. The screenshot of the image editing tool is shown in Figure 2.

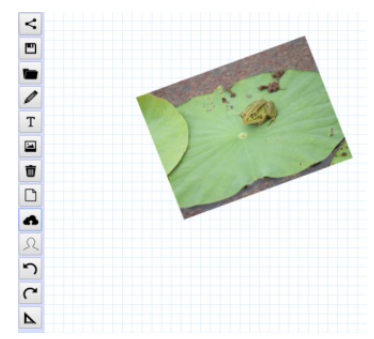

Figure 2. The Screenshot of The Image Editing Tool

The collage creator can arrange the image-materials on canvas and also perform basic image processing (resize, rotation, layer management). The tool can output the created collage image and a XML file in which the procedures and the parameters of the image processing are described. In this time, the collage creator submits the collage image and the XML file to the collage analyst. After that, the collage analyst converts the XML file to the SQL file, and stores the collage image and the image processing information to each table in the collage database. Additionally, collection of the image-material is selected from the web $[11,12,13]$ by the administrator of this system.

\subsection{An Analysis Method Of Appropriate Combination Of The Image- Materials And The Image Processing For The Collage Image}

We show an analysis method of appropriate combination of the image-materials and the image processing for making a collage image. 
The International Journal of Multimedia \& Its Applications (IJMA) Vol.9, No.1, February 2017

\section{Step 1. Choosing Specific Collage Images in The Collage Image Table}

For example, when the collage creator produces a collage image representative of 'funny', the data where the 'c_imp' attribute of the collage image table is 'funny' is extracted by the system. Then, the extracted data is defined as a new table on the database by using The view function of SQL.

\section{Step 2. Sorting The Image-Materials Obtained by Step 1 into The Frequency Order and Extracting Top 3 of The Image-Materials}

There are attributes of 'm_id' and 'c_id' on the image processing table on the image database. The collage image 'c_id' and the used image-materials ' $m \_i d$ ' are related by this table. The image-materials with high frequency are searched by the ' $m$ _id' attribute defined in Step 1. In this time, we extract top 3 of the image-materials based on the results of several experiments.

\section{Step 3. Outputting Combination of Manipulations and Parameters of Three Image- Materials}

After finishing 3 steps, characteristic impression is extracted on the specific collage image.

\section{EXPERIMENTS AND DISCUSSION}

In the experiments, while following the steps to analyze the impression of the image-material, we had carried out a questionnaire survey to 31 persons ( 12 males and 19 females) to set numerical values of four impression words ('warm', 'dynamic', 'happy', 'light') of each image-material. The most frequent value on the results of the questionnaire were adopted as numerical values of each impression word. The screen of the questionnaire is shown in Figure 3. And also, the imagematerial $\mathrm{A}$ is shown in figure 4. The data of the impression word for the image-material $\mathrm{A}$ is shown in Table 4. In the results of the questionnaire survey for the image-material, the most frequent value of 'light' was -2. In this reason, the image-material A is regarded as 'heavy'.

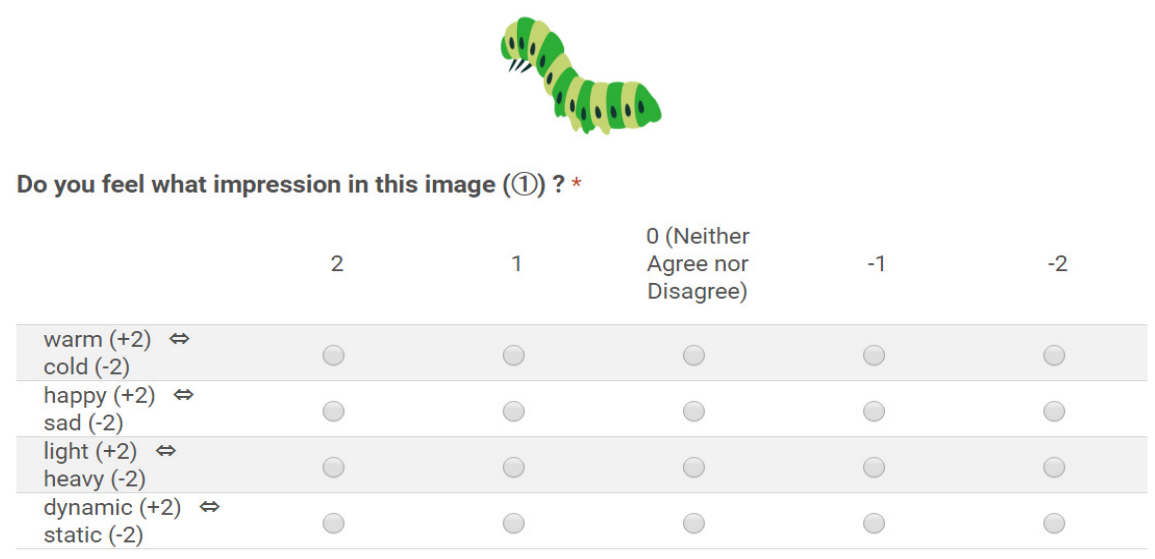

Figure 3.The Screenshot of The Questionnaire 
The International Journal of Multimedia \& Its Applications (IJMA) Vol.9, No.1, February 2017

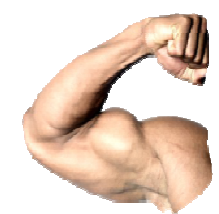

Figure 4.The Image-Material A

Table 4. The Numerical Value and Impression Word in The Image-Material A

\begin{tabular}{|l|c|}
\hline Impression & Value \\
\hline warm & 1 \\
\hline dynamic & 2 \\
\hline happy & 1 \\
\hline light & -2 \\
\hline
\end{tabular}

After finishing the questionnaire survey, we had 20 creators (11 males and 9 females) created collage images having impression of 'funny' as a fundamental experiment in order to analyze relevance between the image and the impression. We also analyzed following points and considered about representation of 'funny'.

- What kind of impression and image-materials of the genre does a collage creator?

- What kind of the image processing is operated the image-materials by a collage creator to make a collage work?

The results of the experiment are shown in table 5. Figure5. and 6.are collage image that the creators actually created at the expreiments. The values of top five of each impression word that is frequently used were as follows: 'human' and 'animal' were used for the genre of the imagematerials used for the collage images. And also, the image processing used by the collage creators most was 'rotation' in this time.

Table 5. The Results of The Experiment

\begin{tabular}{|c|c|c|c|c|c|}
\hline $\begin{array}{c}\text { Ranking of Usage } \\
\text { Frequency }\end{array}$ & $\begin{array}{c}\text { Genre of Image- } \\
\text { Material }\end{array}$ & warm & dynamic & happy & light \\
\hline $\begin{array}{c}\text { 1st } \\
\text { (17 times) }\end{array}$ & 'human' & 2 & 1 & 1 & 1 \\
\hline $\begin{array}{c}\text { 2nd } \\
\text { (13 times) }\end{array}$ & 'animal' & 1 & -1 & 1 & 1 \\
\hline $\begin{array}{c}3 \mathrm{rd} \\
\text { (11 times) }\end{array}$ & 'animal' & -1 & -2 & -1 & -1 \\
\hline 3rd & 'food' & 2 & -1 & 2 & 0 \\
\hline 3rd & 'symbol' & -2 & 0 & -2 & 2 \\
\hline
\end{tabular}

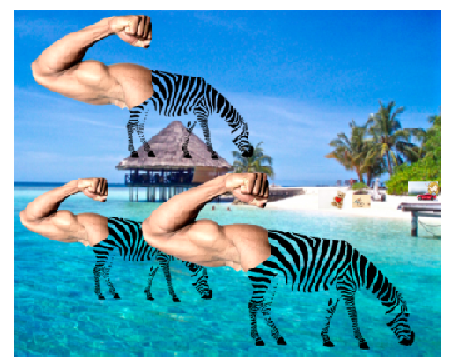

Figure 5. The Collage Image 1 
The International Journal of Multimedia \& Its Applications (IJMA) Vol.9, No.1, February 2017

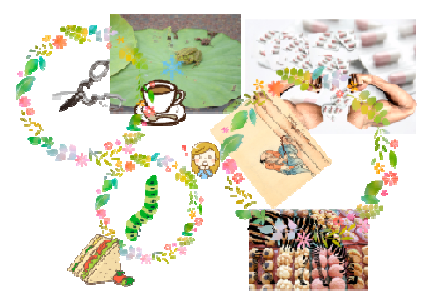

Figure 6. The Collage Image 2

Therefore, we noticed following conditions by the experiments. For making 'funny' collage images,

1. The genre of image-materials is 'human' or 'animal'.

2. The impression of an image-material is that 'warm' is positive value and 'dynamic' has negative value.

3. The same image-material is used in collage image many times.

4. A collage creator turns each image-material in the collage image appropriately.

\section{Conclustion And Future Work}

In this paper, we proposed a new system to support the creators of collage works to find a new expression method by providing an environment to analyze the impression of collage works.

In the collage database in our system, several kinds of collage works created by various human "Kansei", and the numerical values of impression metadata of each collage work. By our system, users and analyzers are able to discover a kind of "collective knowledge" in the collage creation. In this sense, our system leads to a new supporting environment for collage creators and analyzers by providing several new expression methods of collage from this collective knowledge database. In this study, we performed a series of experiments under the following conditions, and examined the feasibility of our system. We set "interesting" as an input impression of collage, 'nature', 'animal', 'food', 'human' and 'symbol' as image genre, 'warm', 'dynamic', 'happy' and 'light' as impressions of the image-materials. We will continue to examine the effectiveness of our system based on the experimental results presented in this study.

Our system seems to have a wide applicability in various academic, art, and psychological field. First, our system has an applicability in the field of art and psychological therapy, because our system is able to collect and archive various collage-work data, and provide various "Kansei"based expression of collage work. In the collage therapy, it is pointed that making a patient create collage works using unusual and unfamiliar materials and arrangement give a good effect to the patient by recognizing the self-understanding and changing the mental situation. Our system gives the new environment to the user (the creator or the patient) to challenge to find a new expression and a human creativity. Second, our system has an applicability in the field of the image retrieval by the following two points: (1) the creation of new images by the combination of multiple images, and (2) the interpretation of the creator's "Kansei" and the intention from the creation process and the newly-created image. In the field of the image retrieval, (2) is not achieved yet.

In our research, as feature work, we focus on the following three points to make our system practical: (1) automatic extraction of the most important expression way in each collage work, (2) database design to correspond to various and heterogeneous expressions of collage work, and (3) detailed checking of the genre and impression-words for collage material images. Our ultimate goal is to get to the essence of psychological situation of human and the rule of "Kansei". To achieve this goal, we have tried to collect the vast amount of real collage data and analyze them quantitatively and deeply. 
The International Journal of Multimedia \& Its Applications (IJMA) Vol.9, No.1, February 2017

\section{REFERENCES}

[1] H.B. Landgarten, "Magazine Photo Collage: A Multicultural Assessment and Treatment Technique," London: Routledge, 1993.

[2] J.W. Stallings, "Collage as a Therapeutic Modality for Reminiscence in Patients With Dementia," Journal of the American Art Therapy Association Volume 27, 2010 - Issue 3.

[3] S. Forzoni, M. Perez, A. Martignetti, S.Crispino, "Art therapy with cancer patients during chemotherapy sessions: An analysis of the patients' perception of helpfulness," Palliative and Supportive Care, Volume 8, Issue 1, pp. 41-48, March 2010.

[4] M. Flickner, H. Sawhney, W. Niblack, "Query by image and video content: the QBIC system," Computer 28, Issue: 9,23-32, 1995.

[5] Y. Hayashi, Y Kiyoki, X. Chen, “A Combined Image-Query Creation Method for Expressing User's Intentions with Shape and Color Features in Multiple Digital Images," Information Modeling and Knowledge Bases, Vol. XXII, pp.258-277, May 2011.

[6] Shaw, J. and Fox, E. Combination of Multiple Searches in Proceedings of TREC-3 (April 1995) 105108.

[7] Yoshitaka, A., and Ichikawa, T. A Survey on Content-Based Retrieval for Multimedia Databases, IEEE Transactions on Knowledge and Data Engineering, 11(1), 81-93.

[8] N.Sonoda, A.Kondo, "Relationship between the style of collage and self," Kurume University psychological research No.5, 13-20,2006.

[9] T. Kurita, T. Kato, "Learning of Personal Visual Impression for Image Database Systems," IEEE Xplore, Oct. 1993.

[10] I.Fuse, S.Okabe, K.Makino, "Copyright Education using Manga materials with awareness of the creativity," JSiSE2014, 323-324, 2014.

[11] Wafu-Sozai.com, http://www.wafusozai.com/.(accessed:2016-01-10)

[12] Photo AC, http://www.photo-ac.com/.(accessed:2016-01-10)

[13] Illust AC, http://www.ac-illust.com/ .(accessed:2016-01-10) 